\title{
AN EvidenCE-BASEd REview of HyPERBARIC Oxygen for Children with Cerebral Palsy
}

\begin{abstract}
This paper reviews the evidence in the literature for the use of hypebaric oxygen $(\mathrm{HBO})$ in the treatment of cerebral palsy $(\mathrm{CP})$. To date there are only two published studies on the outcome of $H B O$ administration in $C P$. A pilot study showed significant improvement in gross motor function, but provided a low level of evidence. A recent multicentre, double blind, placebo controlled trial reported similar improvements in both $H B O$ and placebo groups, but no difference between the groups. This study had a high level of evidence. The results suggest that participation in the trial produced clinically important gains in function. The outcome of the study implies that $H B O$ may have a placebo effect in $C P$
\end{abstract}

\section{KEY WORDS: LITERATURE REVIEW / EVIDENCE, HYPERBARIC OXYGEN / CEREBRAL PALSY}

\section{INTRODUCTION}

Cerebral palsy (CP) is "an umbrella term covering a group of non-progressive, but often changing, motor impairment syndromes secondary to lesions or anomalies of the brain arising in the early stages of its development" (Mutch et al, 1992). The main principle underlying the management of $\mathrm{CP}$ is to maximize the remaining "intact" neurological function, and to incorporate treatment gains into activities of daily living.

Traditional interventions for $\mathrm{CP}$ include orthopaedic surgery, neurosurgery, pharmacological agents, physiotherapy, occupational therapy and speech therapy. These modalities have been in use for many years. More recently, hyperbaric oxygen (HBO) has become a widely used intervention, and has rapidly become perceived as an effective form of therapy by parents of children with CP. There are at present seven centres in South Africa alone offering this treatment. HBO has also been popularised in the South African media, by way of television programs, magazine articles and press reports. There is anecdotal evidence of its positive effects on motor function, cognition and speech.

HBO is a medical treatment that uses pure oxygen to alleviate hypoxia at the cellular level. Pure oxygen is pressurised and administered to the patient in a hyperbaric chamber. $\mathrm{HBO}$ is a recognised medical intervention for several conditions, including carbon monoxide poisoning, wounds, and decompression sickness. It is a controversial intervention in neurological dysfunction, due to the paucity of scientific evidence of its effects on brain functioning.

\section{LITERATURE REVIEW}

HBO has been used since the 1950's to treat a wide variety of neurological conditions, including spinal cord lesions, multiple sclerosis, Alzheimer's disease, head injuries and cerebral vascular accidents (Sukoff, 1984).

HBO was recommended by Neubauer and End (1980) as an adjunct therapy in the treatment of strokes. They stated that it could control cerebral oedema, and provide oxygen to the infarcted and marginally ischaemic areas of the brain. They reported functional gains in their patients after $\mathrm{HBO}$ administration. This was a retrospective before and after study, which provides a low level of evidence (Sackett, 1997). Astrup et al (1981) wrote an editorial in Stroke, postulating the theory that there is a viable area around the cerebral infarct, "the ischaemic penumbra" which could be improved by the administration of HBO. Neubauer wrote to the Lancet in 1990 , referring again to a peri-infarctional zone of viable, but non-functional "idling neurons", which could possibly become functional again by increasing oxygenation of the cerebral blood supply, using $\mathrm{HBO}$.

Enhancement of "idling" neurons, and decrease of cerebral oedema are two of the theories used to explain the effects of $\mathrm{HBO}$ in CP. There is no published scientific evidence to support the supposed improvement of neuronal functioning in $\mathrm{CP}$ by administering $\mathrm{HBO}$.

The first study of HBO in CP came from Brazil. It was presented at a meeting of the Undersea and Hyperbaric Medical Society in 1989 , but was never published and can therefore not be evaluated.

The first research article to be published in this field was a pilot study from Montreal (Montgomery et al, 1999). Twenty-five children with spastic diplegia, aged 3-8 years received $\mathrm{HBO}$ at two treatment centres, 1.5 ATA (atmospheres absolute) for 60 minutes, with a total of 20 treatments. They were assessed before and after the treatment regimen, using a number of tests which included the Gross Motor Function Measure (GMFM), the modified Ashworth scale

CORRESPONDENCE TO:

FM Bischof

PO Box 4105

Germiston South 141 I

Gauteng

Tel: (011) 827-6465 (H) (011) 488-3450 (W) 
for the measurement of spasticity, and video analysis. The improvements in three dimensions of the GMFM were statistically significant. The GMFM is a validated assessment tool with proven reliability (Nordmark et al, 1997). It measures functional limitations, and reduction of these limitations is an important outcome. However, this study involved small numbers, and there were no controls, therefore it would receive the lowest rating on the evidence scales (Butler et al, 1999).

At the time this study was undertaken the Quebec College of Medicine did not approve the prescription of $\mathrm{HBO}$ for children with CP. The results of the pilot study, and pressure from parents of $\mathrm{CP}$ children who wanted this form of treatment recognised by the medical authorities, lead to the design and implementation of a randomised, controlled double blind multicentre trial, which received ethical approval. Five HBO centres participated in the trial, which was conducted by Collet et al and published in the Lancet (2001).

One hundred and eleven children with $\mathrm{CP}$, aged $3-12$ years, were randomly assigned to receive either $\mathrm{HBO}(\mathrm{n}=57)$ or slightly pressurised air $(n=54)$. The HBO pressure was 1.75 ATA and the air pressure 1.3 ATA. This was the lowest pressure at which air could be felt, thus ensuring masking. Forty treatments, each lasting one hour, were given over a period of eight weeks. Children were assessed at baseline, after 20 and 40 treatments, and 12 weeks after cessation of the intervention. The main outcome measures used were:

1. The GMFM, for motor function.

2. The PEDI (Paediatric Evaluation of Disability Inventory), for activities of daily living.

3. Speech and language assessments.

4. Tests for memory and attention.

Therapists were thoroughly trained in the administration of the tests, and blinded to the child's group allocation. Significant improvements for all measures were recorded when comparing pre and post intervention scores. There was however no significant difference between the $\mathrm{HBO}$ and placebo groups in the amount of improvement. This showed that $\mathrm{HBO}$ and slightly pressurised air had the same effect.

The authors state that it could be postulated that air at 1.3 ATA is sufficient to produce the same effect as oxygen at 1.75 ATA, but this does not support the penumbra theory. A possible learning effect was discounted, due to the specific composition and administration of the tests. They concluded that the improvements were probably due to the participation effect. The parents who took part in the study were all highly motivated and spent time with their children in an enriched environment. The outcome of the research implies that $\mathrm{HBO}$ may have a placebo effect in CP.

This was a well designed study. Using the guidelines provided by Guyatt et al (1993), it can be stated that the criteria for validity were met in the conduct of the study. Despite the difficulty in matching participants, due to the heterogenity of $\mathrm{CP}$, there was a good similarity in the distribution of types of $\mathrm{CP}$ between the two groups. Strict inclusion and exclusion criteria were applied.

There was random assignment of the children to either a hyperbaric oxygen, or a pressurised air intervention. Both investigators and participants were blinded to the treatment being administered. The drop out rate was low only 4 children did not complete the study. Both groups were treated equally e.g. physiotherapy was discontinued from 6 weeks before the trial until the end of the treatment period.

Outcomes were measured in all dimensions of the ICIDH 2 (WHO, 1997), namely impairments, functional limitations and societal participation, using validated assessment tools. This study would be placed at Level 1 (the highest level), according to Sackett's method for grading research evidence.

The significant improvements in function obtained by participation in the study have wider implications for other therapies where much time and attention is devoted to children with cerebral palsy, and their parents. The unproven scientific rationale for the use of $\mathrm{HBO}$ in the treatment of children with $\mathrm{CP}$, and the outcome of the controlled study, do not support the recommendation of this expensive intervention.

\section{REFERENCES}

Butler C, Chambers H, Goldstein M, Harris S, Leach J, Campbell S, Adams R, Darrah J 1999 Evaluating research in developmental disabilities: a conceptual framework for reviewing treatment outcomes. Developmental Medicine and Child Neurology 41:55-59

Collet J, Vanasse $M$, Marois P. Amar M, Goldberg J, Lambert J, Lassonde M, Hardy P, Fortin J, Tremelay S, Montgomery D, Lacroix J, Robinson R, Majnemer A 2001 Hyperbaric oxygen for children with cerebral palsy: a randomised multicentre trial. The Lancet 357 : $582-586$

Guyatt GH, Sackett DL, Look DJ 1993 User's guide to the medical literature. Il How to use an article about therapy or prevention. A. Are the results of the study valid? Journal of the American Medical Association 270: 2598-2601

Montgomery D, Goldberg J, Amar M, Lacroix J, Lecomte J, Lambert J, Vanasse M, Marois P The effects of hyperbaric oxygen therapy on children with spastic diplegic cerebral palsy: a pilot project. Undersea Hyperbaric Medicine 26:235-242

Mutch L, Alberman E, Hagberg B, Kodoma K, Velickovic Perat M 1992 Cerebral palsy epidemiology: where are we now, and where are we going? Developmental Medicine and Child Neurology 34:547-555

Neubauer R, End E 1980 Hyperbaric oxygenation as an adjunct therapy in strokes due to thrombosis - a review of 122 patients. Stroke II: $297-300$

Neubauer RA, Gottlich SF, Kagan RL 1990 Enhancing idling neurons The Lancet 335: 542 (Letter)

Nordmark E, Hagglund G, Jarnio GB 1997 Reliability of the gross motor function measure in cerebral palsy. Scandinavian Journal of Rehabilitation Medicine 29:25-28

Sackett Dl, Richardon WS, Rosenberg W, Haynes RB 1997 Evidence-based medicine: how to practice and teach EBM. New York: Churchill Livingstone

Sukoff MH 1984 Update on the use of HBO for the diseases of the central nervous system. Hyperbaric Oxygen Review 5:35-47

World Health Organization 1997 ICIDH-2. International Classification of Impairments, Activities and Participation. Geneva, Switzerland. WHO 